\title{
Fiber Optic Cable Thermal Preparation to Ensure Stable Operation
}

\author{
William J. Thomes, Jr. ${ }^{a}$, Rick F. Chuska ${ }^{a}$, Melanie N. Ott ${ }^{\mathrm{b}}$, Frank V. LaRocca ${ }^{\mathrm{a}}$, \\ Robert C. Switzer ${ }^{a}$, Shawn L. Macmurphy ${ }^{a}$ \\ ${ }^{a}$ MEI Technologies / NASA Goddard Space Flight Center, Lanham, MD, USA 20706 \\ 'NASA Goddard Space Flight Center, Greenbelt, MD, USA 20771
}

\begin{abstract}
Fiber optic cables are widely used in modern systems that must provide stable operation during exposure to changing environmental conditions. For example, a fiber optic cable on a satellite may have to reliably function over a temperature range of $-50^{\circ} \mathrm{C}$ up to $125^{\circ} \mathrm{C}$. While the system requirements for a particular application will dictate the exact method by which the fibers should be prepared, this work will examine multiple ruggedized fibers prepared in different fashions and subjected to thermal qualification testing. The data show that if properly conditioned the fiber cables can provide stable operation, but if done incorrectly, they will have large fluctuations in transmission.
\end{abstract}

Keywords: Space flight, fiber optics, fiber, thermal stability, precondition, NASA, thermal effects

\section{INTRODUCTION}

Fiber optic cables are finding ever-increasing uses in next-generation systems since they possess many advantages over electrical cables: high bandwidth, reduced weight, lower operating power, smaller size, and immunity to electrical interference. These advantages are especially important for flight systems that must be launched into space, but they also play a vital role for ground-based systems and those flown on aircraft. As scientific systems become more advanced, the capability to handle high data rates from multiple sources is essential. Besides handling the necessary functions for controlling a remote platform, such as a satellite, the communications systems must collect data from various sensors and instruments distributed throughout the platform and either act upon those inputs or relay them to external sources. In order for all of these various parts of the optical structure to work together in a predicable fashion, the fiber optic cables must provide stable operation over all environments. Proper preparation of the fiber optic cables will result in a cable that shows little change over the thermal excursions for that mission. However, if the cable construction and conditioning is done incorrectly, the optical transmission through that cable will change dramatically as the temperature changes. With incorrect preparation, the cable transmission will usually continue to worsen with each thermal change until the fiber breaks.

In this paper, we will present fiber optic cable attributes that lead to thermal stability, including the steps for properly preconditioning the cable. First, the method to properly precondition the cable will be discussed. Second, thermal performance of the various properly prepared fiber optic cables will be shown to demonstrate how differences in cable construction can affect optical transmission during changes in temperature. Third, a comparison will be made between properly prepared cables and cables constructed with fibers that were used as received from the manufacturers, without any conditioning. It should be noted that it is beyond the scope of this paper to get into the specifics of fiber optic cabling. Various types of fibers were chosen for this study that represent different types of protective jacketing, as will be explained, but the specifics of exact dimensions and types of protective layers depends heavily on the type of fiber being used and the environment the cable will be subjected to for a given mission.

\section{FIBER OPTIC CABLES AND CONNECTORS}

Six types of fiber optic cables were chosen for this study. Each was chosen because it represents a cable being used in a current system or being considered for an upcoming application. The six cable types are: 
1. TEQS Powerflex hard-polymer coated fiber manufactured by InnovaQuartz, Inc (part number FG365UEC). Fiber size was 365/400/ 425 (TEQS) / 730 (TEFZEL). This was a step-index fused silica core and cladding. This fiber would be representative of a single fiber with no additional cabling, such as would be suitable for routing in a protected area that would not be subjected to outside mechanical interactions.

2. $1.2 \mathrm{~mm}$ Flexlite fiber optic cable manufactured by W.L. Gore. Fiber size was $400 / 440$ step-index fused silica core and cladding. The cable consists of a fiber wrapped in expanded PTFE, Kevlar aramid braid, and a fluoropolymer outer jacket. This cable has been used on multiple NASA space flight missions and is a cabling configuration that provides mechanical strength while allowing for some fiber movement inside the jacketing during thermal changes.

3. Fiber cable used on the International Space Station. The fiber is a 100/140 graded index with a carbon coating. Cabling consists of a Teflon-coated fiberglass braid and an outer polymer jacket. The cable was manufactured by Brand-Rex Company. The cable was designed to provide thermal stability, with the carbon coating acting as a hermetic barrier for the fiber. It was later found that pinholes in the carbon coating can act as enhanced sites for defect growth, so the cable is typically screened before use.

4. Single-mode fiber with a PEEK $1.8 \mathrm{~mm}$ Simplex cabling, manufactured by W.L. Gore (part number GSC-13-83260-00). The inside fiber is a $9 / 125 / 250$ single-mode fiber optimized to work at $1310 \mathrm{~nm}$ but that has also been shown to work well at $1550 \mathrm{~nm}$. The cabling consists of an expanded PTFE, PEEK tubing, aramid strength members, and an outer polymer jacket. The PEEK tube is typically used when additional mechanical strength is needed.

5. Multifiber ribbon cable, manufactured by W. L. Gore. The cable consists of 12 fibers that are 100/140 microns. The fibers are laminated in Mylar with an expanded PTFE wrap and are then covered with a Kevlar braid for strength and coated in an outer polymer jacket. The cable design allows for termination with an MTP connector.

6. Strong Tether Fiber Optic Cable (STFOC), manufactured by Linden Photonics (Cable ID of tested cable is 0609001 ). The fiber is a single-mode $9 / 125$ with an outer protective buffer. A non-kink version is also offered that is supposed to provide additional protection by adding an outer tube layer. Since the outer tube had not yet been tested to meet outgassing requirements for NASA missions, the regular version of the fiber was tested.

All cables, except the multifiber ribbon cable, were terminated with FC connectors, as shown in Figure 1. The FC connector has a $2.5 \mathrm{~mm}$ diameter ferrule that is spring-loaded. A key on the connector provides clocking. A larger outer nut is used to secure the connector into an adapter. An APC (angled) polish was used on the single-mode fibers. 


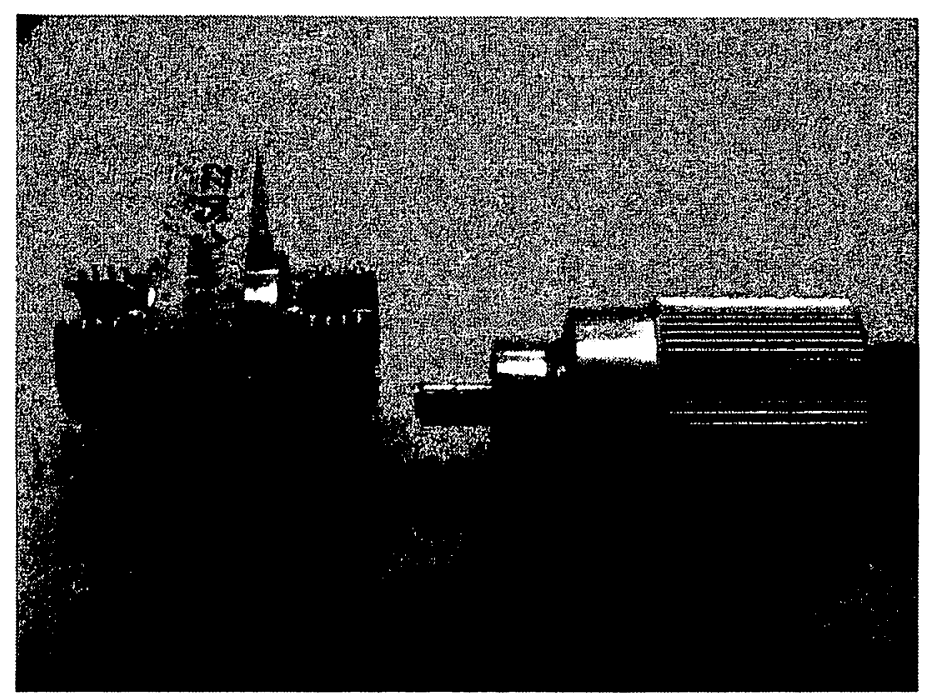

Figure 1: An FC connector and adapter manufactured by Diamond.

During termination of the cables, the strength members were crimped to the back of the connector using a metal crimp sleeve, as shown in

Figure 2. This provides strength to the fiber connector interface and is the common termination method for these types of cables and connectors. Only the TEQS and STFOC cables were not terminated in this manner, since the fibers were not cabled with additional strength member layers. For these two fiber types, the fibers were glued into the ferrule (same was done for all terminations), but no additional attachment was made between the fiber and connector. In all terminations, a Hytrel boot was used to provide support for the fiber behind the connector. The boot fits the back of the connector snugly, but it loosely holds the fiber and will let it slide in and out with little resistance.

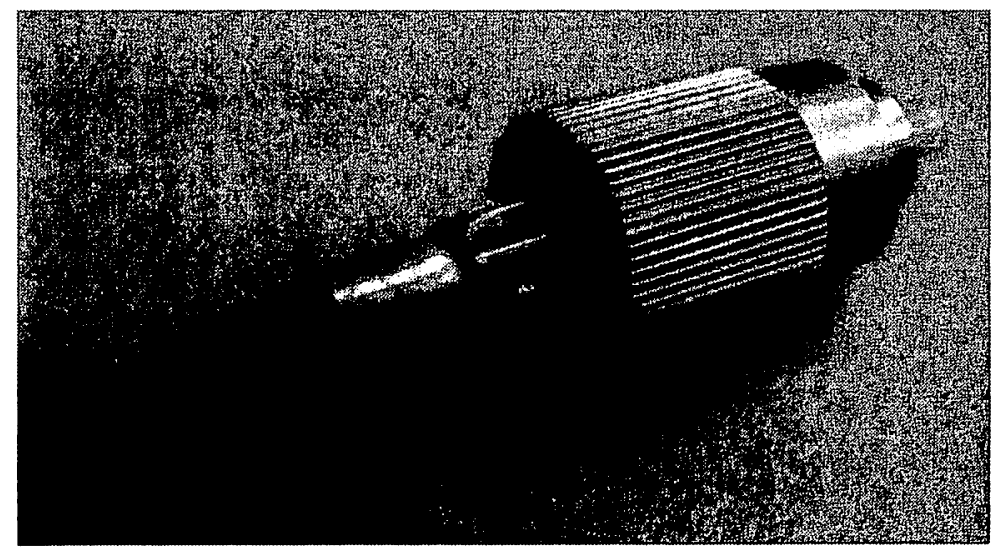

Figure 2: Back of FC connector, showing the metal crimp sleeve used to attach the cable strength members to the connector.

\section{THERMAL PRECONDITIONING}

Many of the materials used in the cabling of fiber optics for protection are either extruded onto the cable or are applied while the cable and coating are not at the same temperature. Thus, there are residual stresses in the cable layers after manufacturing. This alone does not cause a problem for the cable, but when it is subjected to thermal cycling, the cable can shrink to relieve some of the stress. 
For this part of the study, we took samples of each type of fiber optic cable and subjected them to thermal cycling and measured the change in length at fixed intervals during the test. Three samples of various lengths were chosen: 1,3 , and 6 meters for all samples, except the STFOC fiber, for which only one $6 \mathrm{~m}$ sample was studied (due to the limited amount of that cable). The different lengths allow for comparing the effect of cable shrinkage at the end of the cable against the change in length along the middle portion of the cable.

The preconditioning temperatures were chosen based on a space flight mission requirement of $-50{ }^{\circ} \mathrm{C}$ to $125^{\circ} \mathrm{C}$, such as would be needed for the International Space Station. A general rule of thumb is to add between $5{ }^{\circ} \mathrm{C}$ and $10^{\circ} \mathrm{C}$ to the upper survival temperature requirement. It should be noted that the precondition temperatures should be determined by the survival temperature requirements rather than by the operating requirements because even though the fiber optic cable will not need to be operating during the survival temperatures, the cable will shrink and stress the connectors if it was not preconditioned to that range. The residual stress will then lead to unstable performance even over the operational temperature range. A rule of thumb for the lower temperature is to use either the lower survival temperature limit or $-30^{\circ} \mathrm{C}$, whichever is less. Temperatures lower than $-30^{\circ} \mathrm{C}$ do not cause appreciable rearrangement of the material structures for typical cabling materials. So for most materials, this lower limit is sufficient and will reduce the time needed to precondition the cables.

Based on the selected survival temperatures, the thermal preconditioning of the fiber optic cables in this study was carried out from $-30^{\circ} \mathrm{C}$ to $130^{\circ} \mathrm{C}$, with a ramp rate of $2{ }^{\circ} \mathrm{C} / \mathrm{min}$ and a dwell of 1 hour at the hot and cold extremes. Figure 3 shows a typical temperature profile used for preconditioning of the fibers.

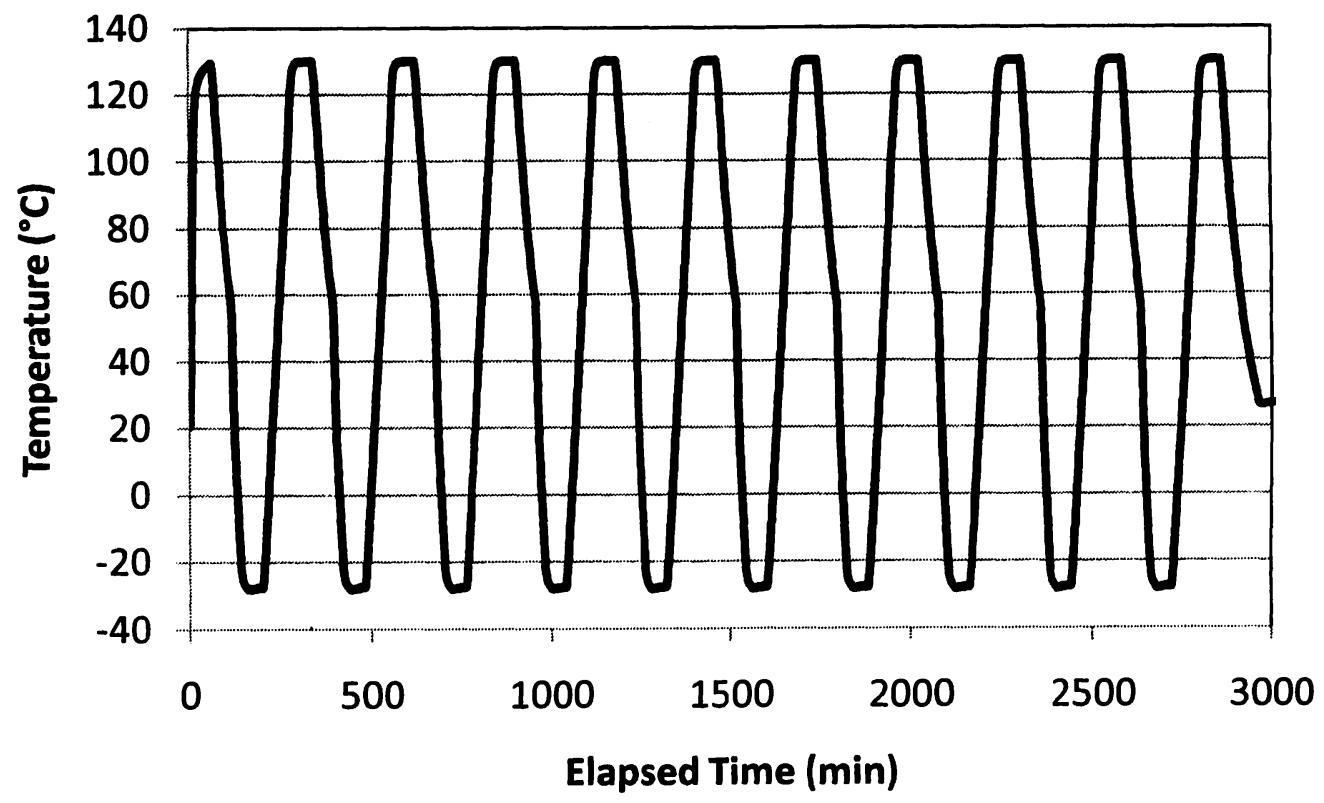

Figure 3: Temperature profile for fiber optic cable preconditioning.

The fiber cables were removed from the thermal chamber after $5,10,15,20,30,40,50,60,70$, and 80 cycles and measured. The multifiber ribbon cables and SMF-28 PEEK cables were subjected to an additional 20 cycles, for a cumulative total of 100 cycles.

Figure 4 shows the percentage change in cable length with thermal cycling. Almost all cables showed some shrinkage during the preconditioning. Only the STFOC and TEQS coated fibers showed almost no change in length. This change in cable length that eventually saturates (the cable does not shrink further with additional cycling) is used to determine whether the preconditioning has been effective, as well as the number of thermal cycles needed to ensure that the cables will exhibit stable operation during deployment. Also, the data show that in general, shorter cables show a higher percentage change in length than longer 
cables. While the percentage change in cable length is useful for observing the saturation behavior of the cable shrinkage (and is how the data are usually presented), the actual change in cable length at the end of the cable, where it attaches to the connector, needs to be considered also.

Aside from the inherent thermal stability of a fiber optic cable due to the material coefficients of thermal expansion matches for the various layers, the transmission performance over changing thermal environments will be dictated in a large part by any stresses on the fibers at the connectors due to cable length changes. If the cable shrinks with thermal cycling after it has been terminated, the easiest place for it to relieve stress is inside or immediately behind the fiber connector, since this is where the outer protective layers have been removed to install the connector.

Figure 5 shows the overall change in cable length with thermal preconditioning cycles for all of the cables under study. Similar to the percentage change in cable length, there is a large initial change that saturates with further cycling. As can be seen from the data, longer cables exhibit a larger change in cable length. Also, different cable constructions require different numbers of thermal cycles to reach a stable length.

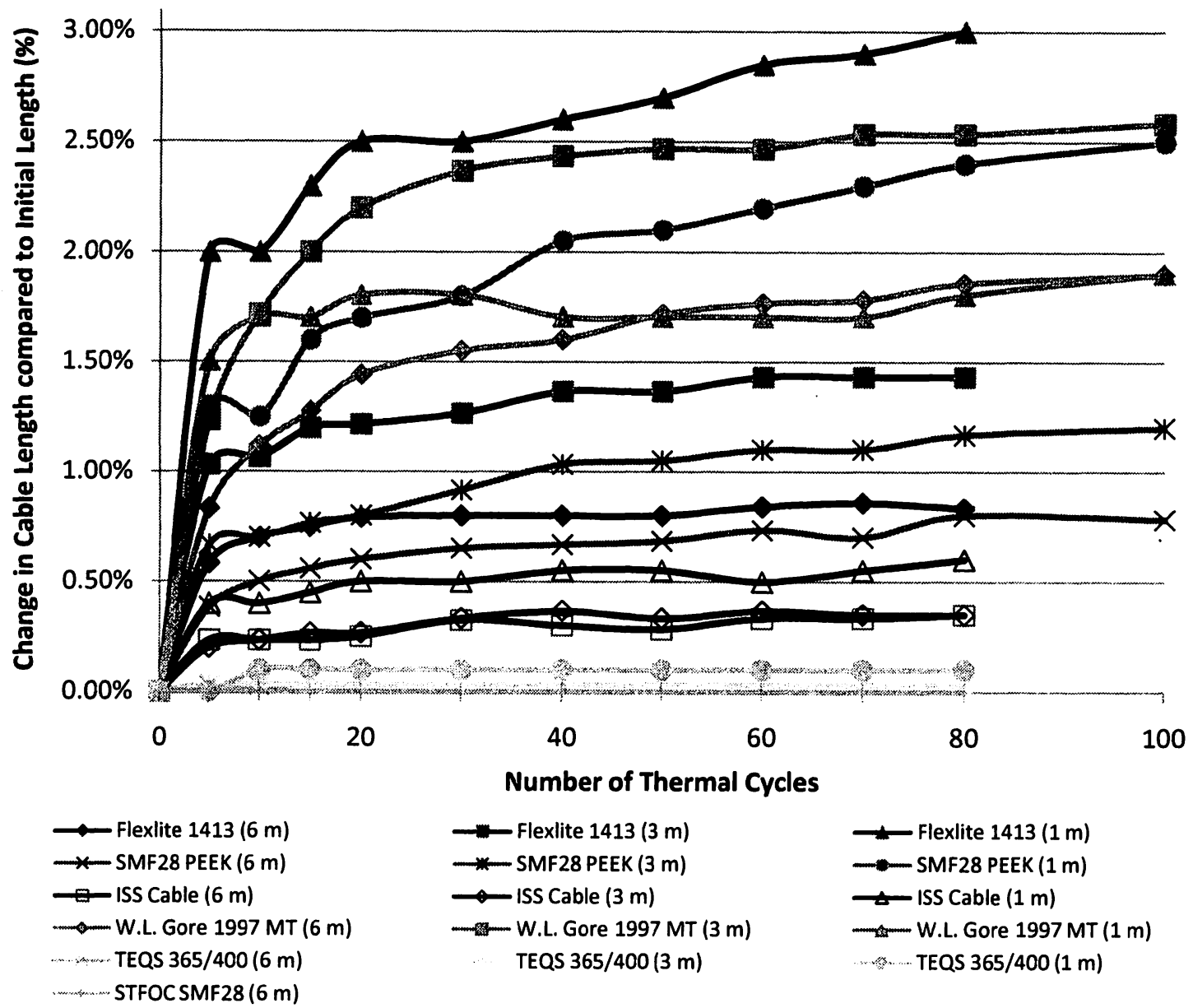

Figure 4: Percentage change in cable length with thermal preconditioning cycles. 


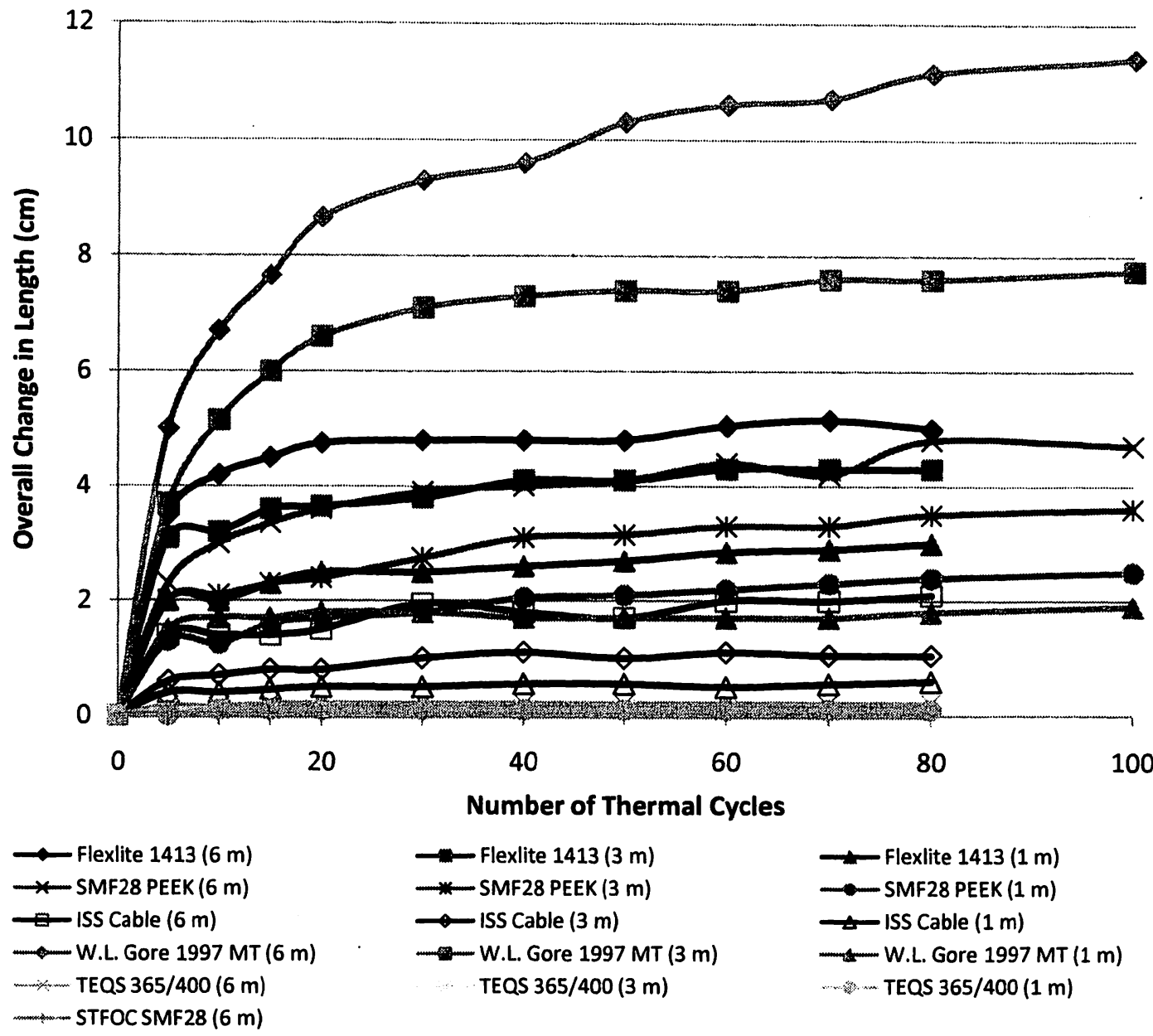

Figure 5: Overall change in cable length with thermal preconditioning.

Comparing the data presented in Figure 4 and

Figure 5 shows that the longer the cable, the larger the overall length change, but the smaller the percentage change in cable length. This means that the majority of the cable shrinkage occurs near the end of the cable and that the middle portion of the cable has a smaller effect on the change in length. This is extremely important for preconditioning fiber optic cables, especially those that show a larger overall length change, because the cables must be cut close to final length before preconditioning. If the cable is properly preconditioned and then cut shorter, it will shrink further with subsequent thermal cycling. For applications requiring tighter tolerance on the cable length, the shrinkage during preconditioning must be taken into account before the cables are first cut.

In addition to removing manufacturing-related residual stresses from the fiber cables, thermal preconditioning is also a good incoming inspection step for the fiber optic cables. Oftentimes, minor manufacturing defects will become exaggerated during thermal cycling. Issues such as poor adhesion of coatings or imperfections in extruded polymers will lead to visible changes in the cable following preconditioning, and these cables can then be screened out early in the manufacturing and testing process. So even cables that show little or no shrinkage, like the TEQS and STFOC, can benefit from some degree of thermal preconditioning.

As a final note on proper preconditioning of fiber optic cables, the thermal profile should include cycling of the temperatures from hot to cold. This cycling is important for allowing the materials in the various layers 
to reorient their structural bonds to achieve a lower energy and often denser configuration. If the temperature is increased or decreased and just held longer, it will not replace cycling the cable from a hot to a cold temperature.

\section{OPTICAL PERFORMANCE OF PROPERLY PRECONDITIONED CABLES}

Once properly preconditioned, fiber optic cables should exhibit relatively minor fluctuations in optical transmission over thermal environments. Typical values are $0.2 \mathrm{~dB} / \mathrm{m}$ or less for single-fiber cables. However, external stresses on the fiber can increase this value.

To examine the change in optical transmission of the fiber optic cables over temperature, the insertion loss change was monitored while the cables were subjected to thermal cycling from $-50^{\circ} \mathrm{C}$ to $125^{\circ} \mathrm{C}$ at a ramp rate of $2{ }^{\circ} \mathrm{C} / \mathrm{min}$ and a dwell of $1 \mathrm{hr}$ at the hot and cold extremes (this is the same profile used for determining the correct preconditioning temperatures in the previous section).

Figure 6 shows the experimental setup. An optical source (Rifocus 752L dual LED source for either 850 $\mathrm{nm}$ or $1310 \mathrm{~nm}$ or a Santec tunable source set to $1550 \mathrm{~nm}$ ) was coupled to a splitter. Outputs from the splitter were connected to the samples (connectors outside the thermal chamber for this test) and one splitter output was connected directly to the optical detectors for monitoring source power during the test. The other ends of the samples were connected directly to the optical detectors. Both $850 \mathrm{~nm}$ and $1310 \mathrm{~nm}$ were used with the multimode fibers being tested, but $1550 \mathrm{~nm}$ was used for testing all of the single-mode fibers (PEEK and STFOC).

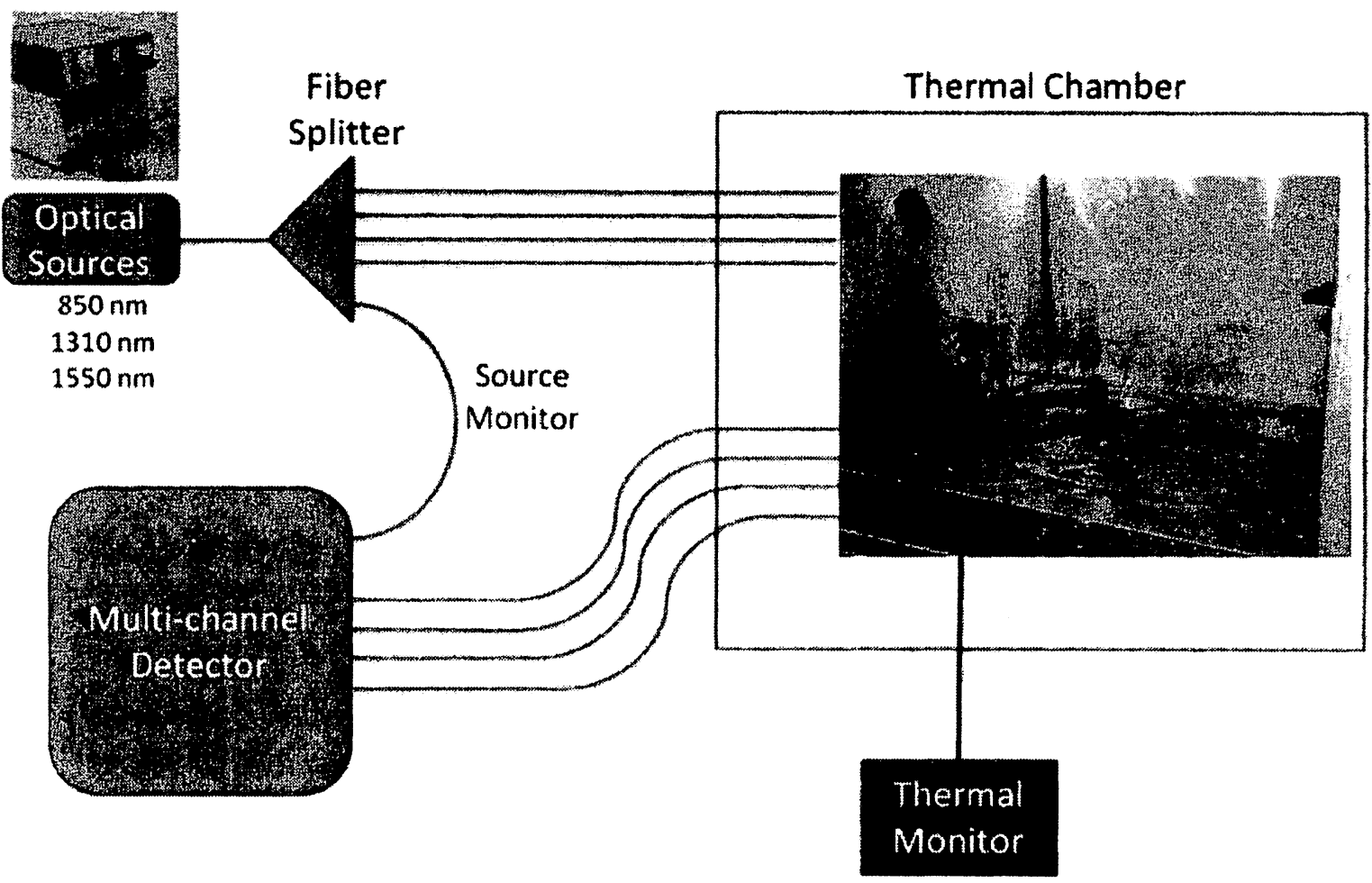

Figure 6: Experimental setup used for active monitoring of insertion loss for thermal cycle testing of the fiber optic cables.

Figure 7 shows the change in insertion loss at $850 \mathrm{~nm}$ for the Flexlite and TEQS coated fibers. The insertion loss has been normalized for length. From the data, it can be seen that the samples show less than $0.2 \mathrm{~dB} / \mathrm{m}$ change in insertion loss during the thermal cycling. It should be noted that the shorter samples are more susceptible to thermal gradients during the transition into and out of the thermal chamber. Therefore, they will show a slightly higher loss when normalized to length because of the larger 
contribution from stresses in this transition region. For extrapolating the data to a system configuration or to longer cables, the insertion loss change after the first set of cycles should be used. Taking this into account, the TEQS fibers show thermal effects of $0.02 \mathrm{~dB} / \mathrm{m}$ or less, and the Flexlite fibers are closer to $0.12 \mathrm{~dB} / \mathrm{m}$ over this thermal cycling range.

Figure 8 shows the same analysis for the PEEK, STFOC, and ISS cables. All of these cables show insertion loss changes comparable to the TEQS coated fiber. The jumps in the insertion loss are an artifact of the measurement system. Due to the higher transmission of the single-mode fiber at $1550 \mathrm{~nm}$, the detector is set to a higher range. Therefore, small changes in the last one or two significant digits result in jumps in the insertion loss on this scale.

Figure 9 shows the thermally induced change in insertion loss for the Flexlite cables at both $850 \mathrm{~nm}$ and $1310 \mathrm{~nm}$. From the chart, the insertion loss is seen to be slightly more sensitive at $850 \mathrm{~nm}$ than $1310 \mathrm{~nm}$. A small wavelength dependence of the thermally induced insertion loss changes is typical, and the fiber type will determine whether longer or shorter wavelengths are more sensitive.

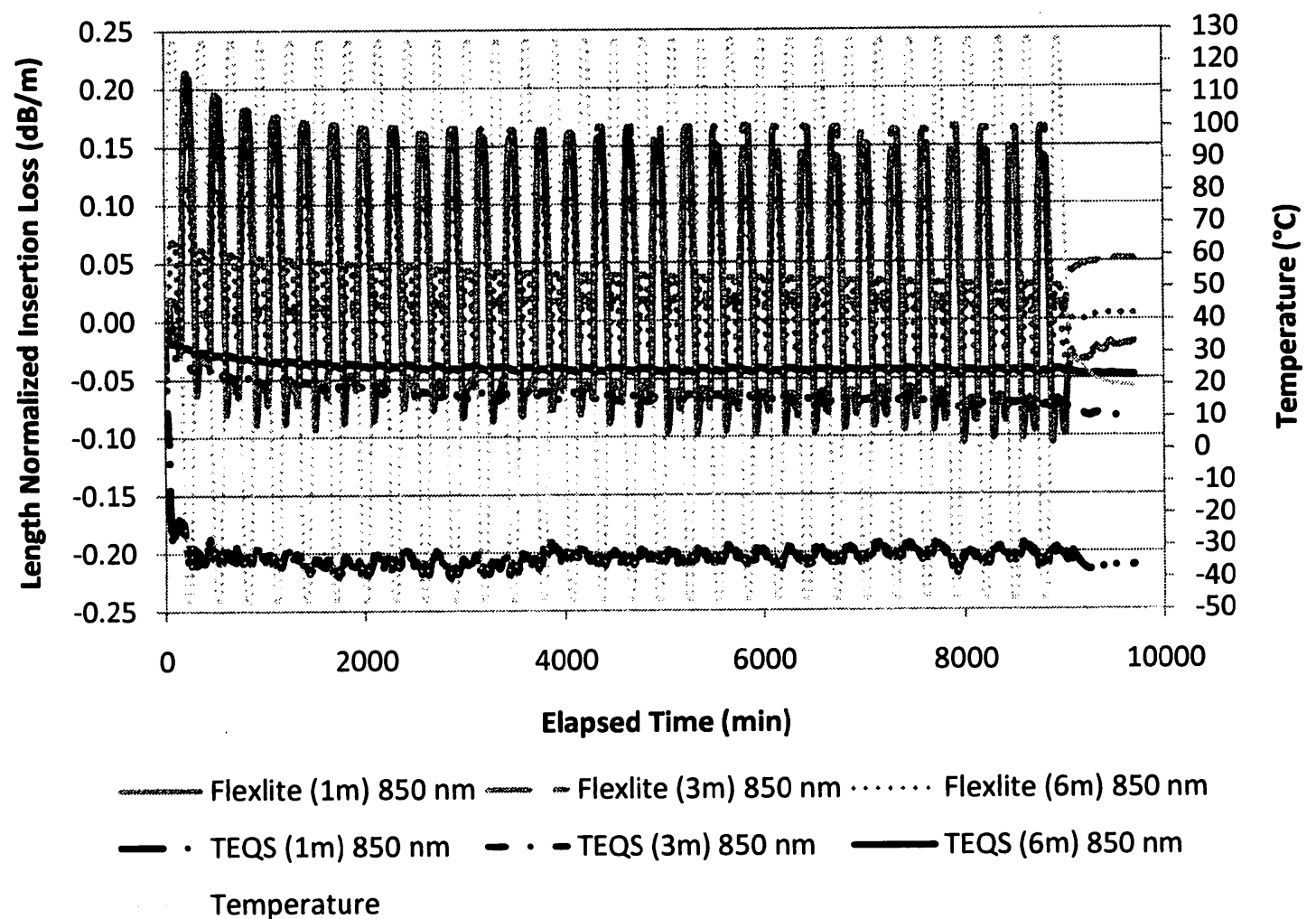

Figure 7: Length normalized insertion loss change with thermal cycling for TEQS and Flexlite fibers. 


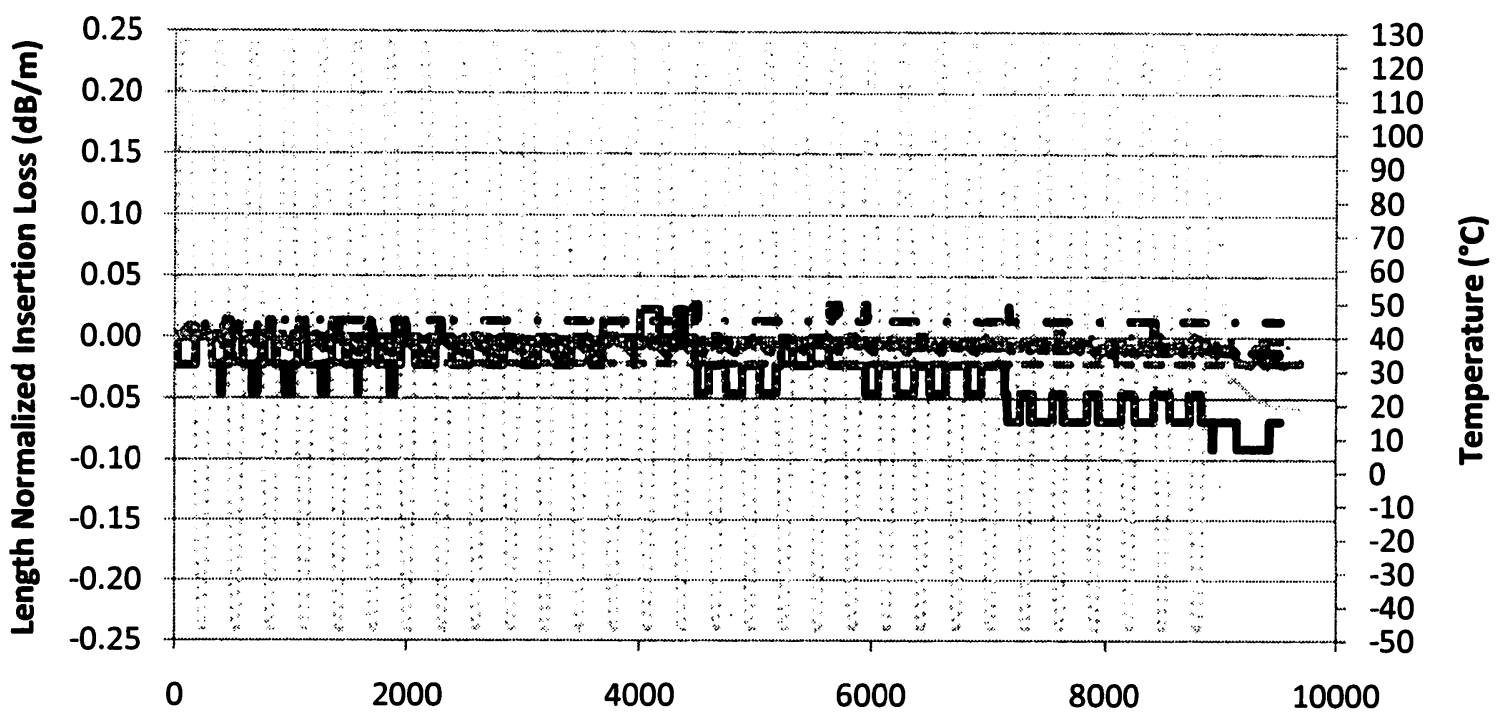

Elapsed Time (min)

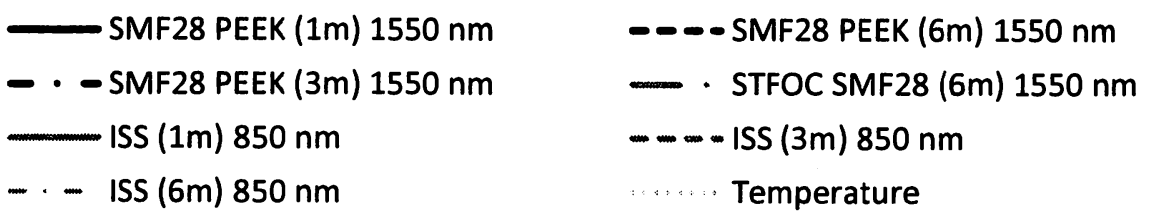

Figure 8: Length normalized insertion loss change with thermal cycling for PEEK, STFOC, and ISS cables.

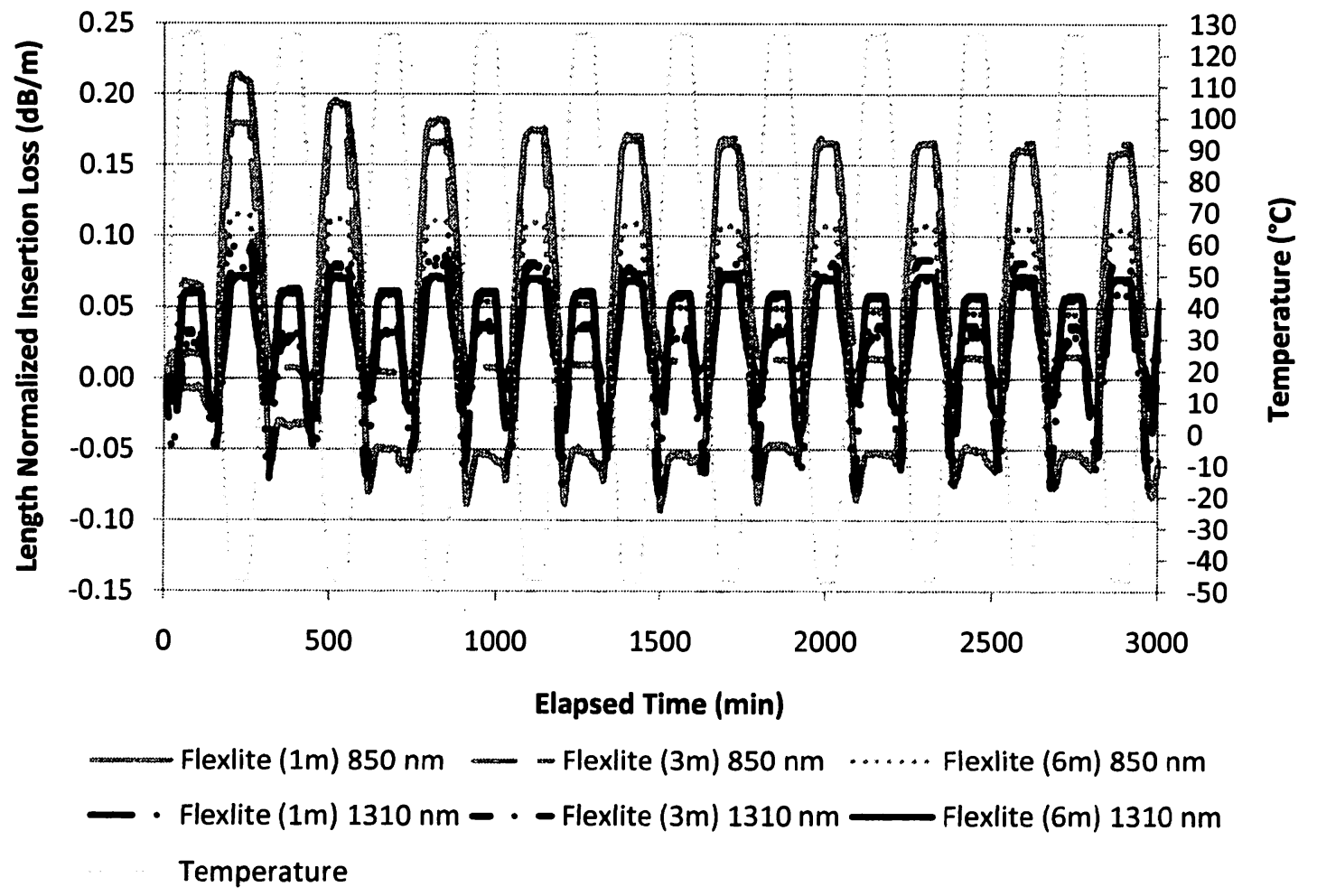

Figure 9: Length normalized insertion loss changes of Flexlite cables with temperature at $850 \mathrm{~nm}$ and $1310 \mathrm{~nm}$. 


\section{PRECONDITIONED VERSUS NONPRECONDITIONED FIBER PERFORMANCE}

In addition to the fiber optic cables prepared using properly preconditioned cables, cables identical to the 1and 3-meter samples were prepared using nonpreconditioned cables. The different cable types were then subjected to thermal cycling using the same setup as in the previous section; however, in this test, the entire cable and connectors were placed inside the thermal chamber. Optical signals were carried into and out of the thermal chamber using specially prepared optical fibers with no jacketing. These lead-in and lead-out cables were terminated with the appropriate FC connectors for the fibers being tested. Identical thermal profiles of $-50^{\circ} \mathrm{C}$ to $125^{\circ} \mathrm{C}$ with a $2^{\circ} \mathrm{C}$ ramp rate and $1 \mathrm{hr}$ dwell at hot and cold extremes were used while the insertion loss of the cables was being monitored. Testing was also conducted on the lead-in and leadout cables without the samples present to ensure their thermally induced insertion loss changes were negligible in comparison with the insertion loss changes seen in the sample fibers.

Figure 10 shows the change in insertion loss with temperature cycling of the International Space Station preconditioned and nonpreconditioned cables. From the graph, it can be seen that the $1 \mathrm{~m}$ nonpreconditioned sample showed a larger thermally induced insertion loss change than any of the other cables. The $3 \mathrm{~m}$ nonpreconditioned cable exhibited slightly higher insertion loss effects than the preconditioned cables. The negative insertion loss change, indicating higher transmission, for the $3 \mathrm{~m}$ nonpreconditioned cable is likely due to a change in the coupling with the fibers leading into and out of the thermal chamber. This improvement is not typical since stress in the connector would usually lead to degraded coupling between the two fibers, but on occasion a small improvement is measured. However, the insertion loss change with thermal cycling is accurate since the background drift after the first cycle is small in comparison to the thermally-induced changes.

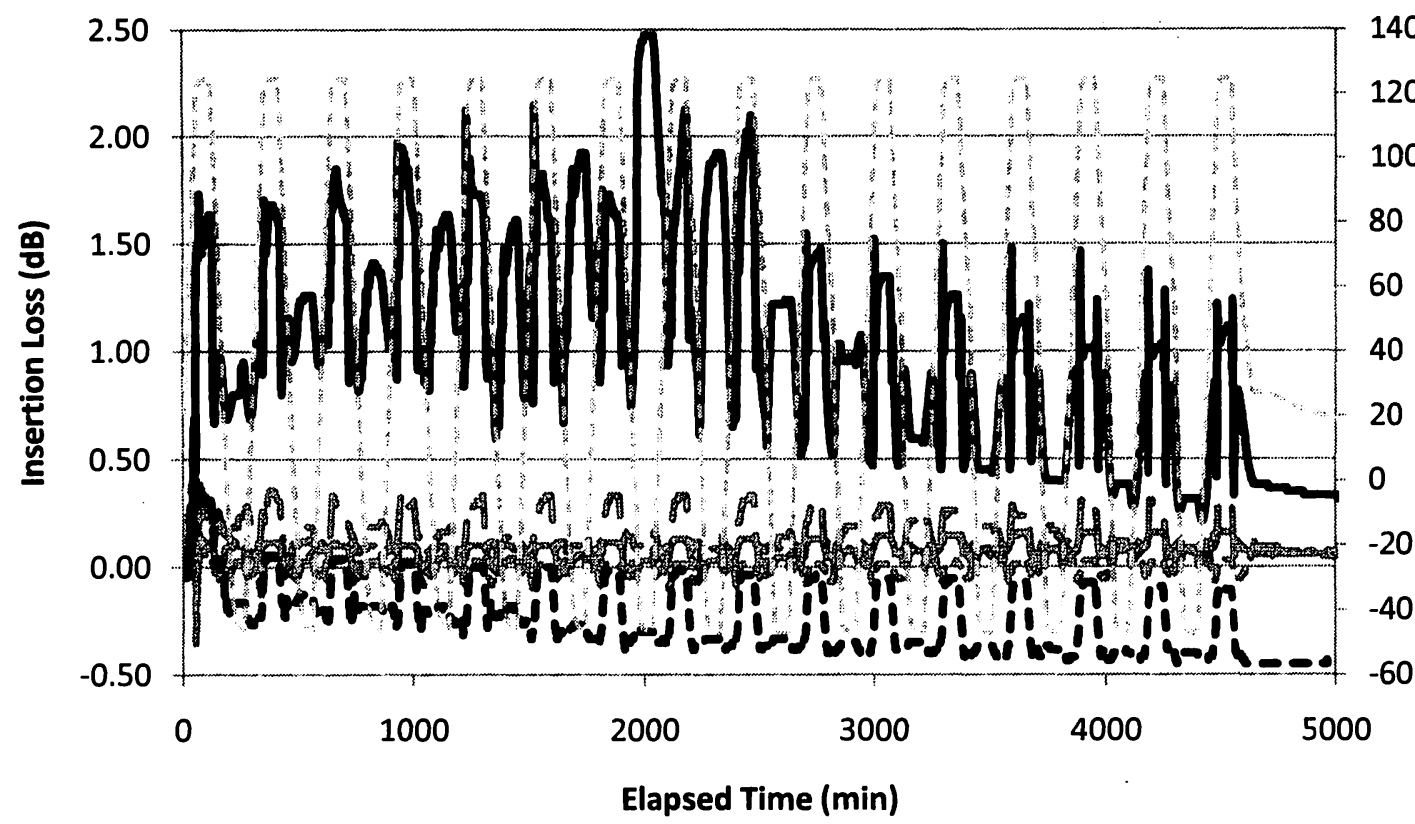


Figure 11 shows the thermally induced change in insertion loss of preconditioned and nonpreconditioned SMF fiber with the PEEK protective tubing cable configuration. The data show that as with the ISS cable, the nonpreconditioned PEEK cables have a larger insertion loss change with thermal cycling, as compared to the preconditioned cables. However, since the PEEK cables shrink more than the ISS cables during preconditioning, the stressing of the fiber in the nonpreconditioned PEEK cables is larger, and thus the insertion loss changes are higher. The $1 \mathrm{~m}$ preconditioned cable also shows a slight increase of insertion loss during the later thermal cycles. This suggests that the cables may not have been preconditioned enough and should have been subjected to more preconditioning cycles. From

Figure 5, it can be seen that the $1 \mathrm{~m}$ PEEK cable was still shrinking slightly with thermal cycling.
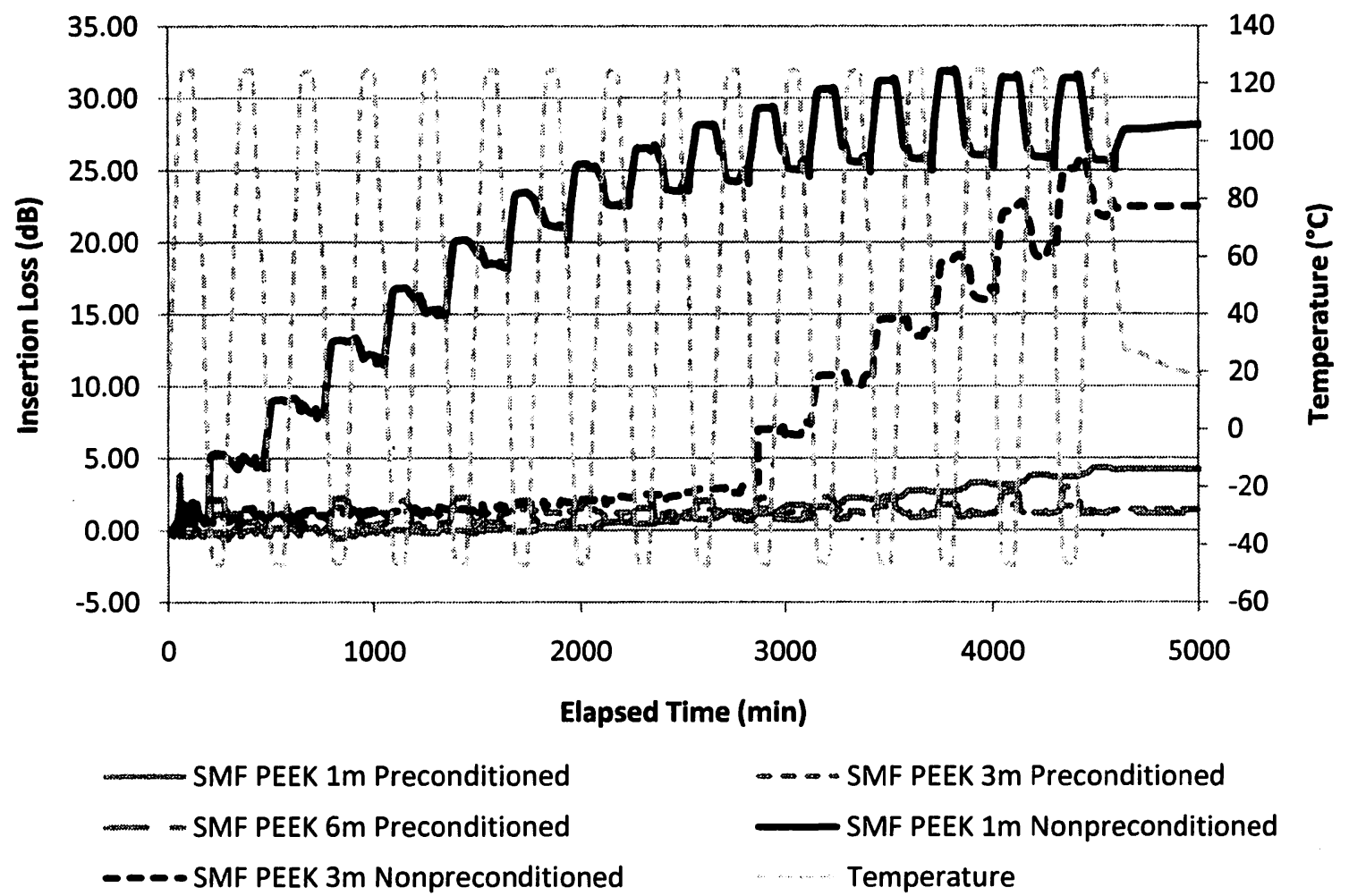

Figure 11: Comparison of insertion loss versus temperature for preconditioned and nonpreconditioned SMF PEEK cables measured at $1550 \mathrm{~nm}$.

Comparison of the insertion loss changes in preconditioned and nonpreconditioned cables shows the improved thermal stability of the cables that resulted from preconditioning the fiber. The effects are most pronounced in the shorter nonpreconditioned fibers because as they start to contract, there is less room along the fiber inside the cabling to accommodate the extra length. The outer jacket and strength members are shrinking while the fiber itself is staying essentially the same length (the CTE change is small compared to the typical shrinkage of the cable materials). The longer cables can accommodate some of the extra fiber length, but they will also eventually start to stress the fiber at the weakest point in the cable, which is almost always where the cable attaches to the fiber connector. This effect explains why the longer cables will sometimes not show a large thermal effect until enough cycles have been completed, at which point the cable will have a large insertion loss change over a few thermal cycles, as with the PEEK cables.

\section{CONCLUSIONS}

The use of fiber optic cables in space flight and other harsh environments requires that their optical transmission be stable over large changes in temperature. In this study, six types of fiber optic cables 
suitable for space flight were studied to determine the proper method of thermally preconditioning the cables, to examine the optical performance of those cables, and to compare properly preconditioned cables against nonpreconditioned cables. All cables chosen for the study, except for the TEQS and STFOC, have passed NASA requirements for flight use, including outgassing. The TEQS fiber represented a hard polymer coated fiber, as would be typical for a protected routing. The STFOC fiber has a harder outer coating, but it was found to be very sensitive to bending. There is a non-kinking version of the STFOC, but it was not included because it has not yet been tested to meet NASA outgassing requirements. Standard commercial off-the-shelf fiber optic cables with a PVC jacket, as would typically be purchased from a large optical supplier, are not suitable for space flight and were therefore also not included in our study.

Length measurements taken at intervals during the preconditioning demonstrate the shrinkage of the cable during thermal cycling. Different fiber cable configurations will shrink by different amounts, but thermal preconditioning should be continued until the change in cable length has stabilized. Comparing the percentage change in length with the overall change in length illustrates the need to cut the fiber optic cable to length before preconditioning, as the cables shrink more near the ends than in the middle.

Optical performance of the various cables was measured by thermally cycling from $-50^{\circ} \mathrm{C}$ to $125^{\circ} \mathrm{C}$. The preconditioned cables were inserted into an oven with the connectors outside the chamber. Thermally induced insertion loss changes of $0.2 \mathrm{~dB} / \mathrm{m}$ or less were observed for all of the different cables. Measurements were taken at multiple wavelengths to demonstrate the slight wavelength dependence of the insertion loss thermal effects, but the exact magnitude of the dependence will depend on the fiber used and the cable construction.

To highlight the importance of proper preconditioning of the fiber optic cables, data were presented for thermal cycling for both preconditioned and nonpreconditioned cables. The nonpreconditioned cables showed a dramatically larger insertion loss fluctuation with temperature when compared to the identical preconditioned cable. Shorter cables were more susceptible to the thermally induced stresses.

\section{ACKNOWLEDGMENTS}

The authors would like to thank the NASA Electronic Parts and Packaging Program (NEPP) for providing funding for this work. In addition, we would like to acknowledge the assistance of W. L. Gore and Linden Photonics for providing samples of various fibers for this study. 